Graphical Abstract

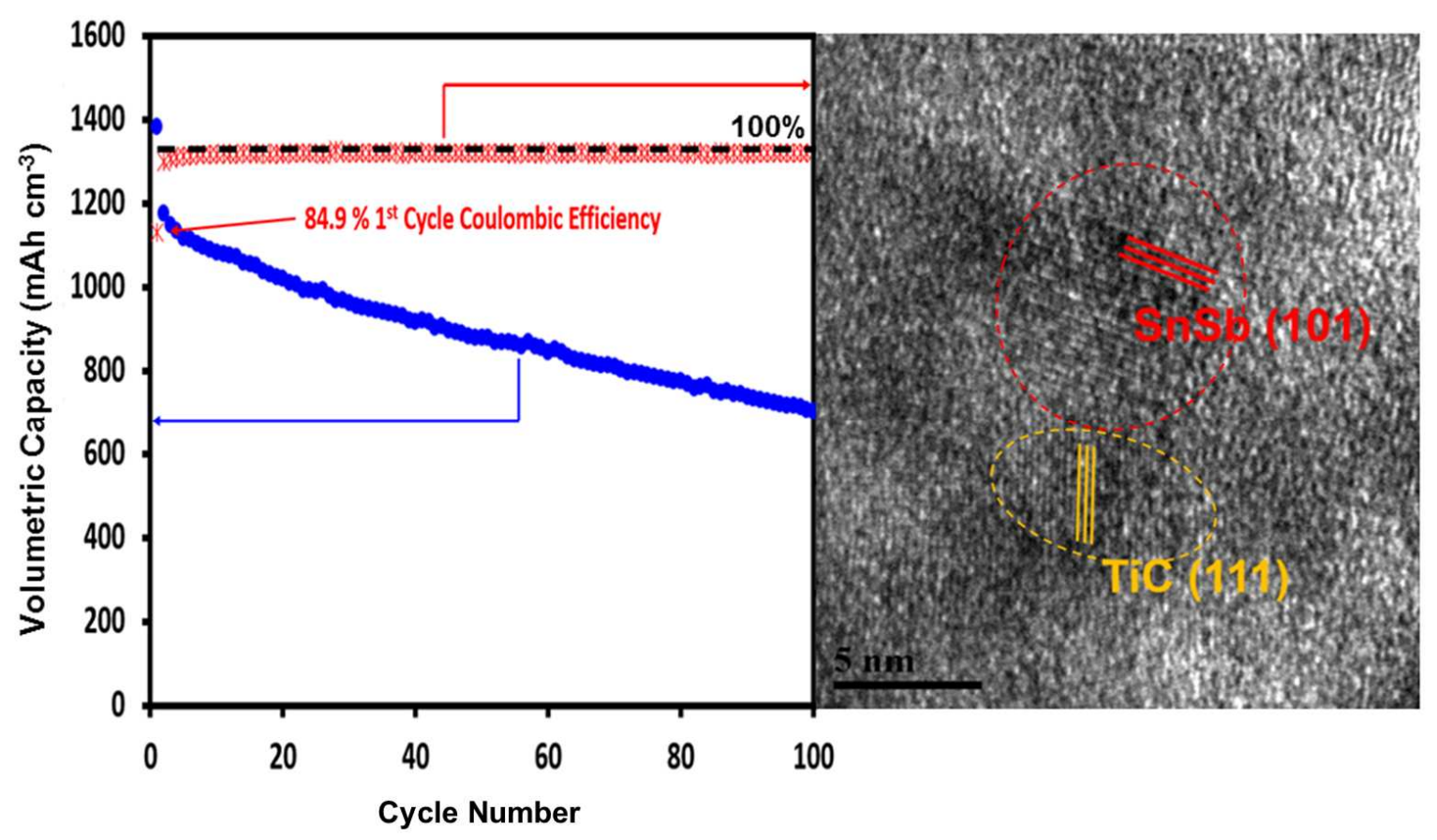




\title{
SnSb-TiC-C nanocomposite alloy anodes for lithium-ion batteries
}

Joshua Leibowitz, Eric Allcorn, and Arumugam Manthiram* Materials Science and Engineering Program and Texas Materials Institute The University of Texas at Austin

Austin, TX 78712, USA

\begin{abstract}
SnSb-TiC-C nanocomposite alloy anodes for lithium-ion batteries have been synthesized by a mechanochemical process involving high-energy mechanical milling of Ti-Sn and Ti-Sb precursors with carbon black. Characterization of the formed nanocomposites using x-ray diffraction (XRD), scanning electron microscopy (SEM), and transmission electron microscopy (TEM) reveals that these alloys are composed of crystalline nanoparticles of $\mathrm{SnSb}$ dispersed in a matrix of crystalline $\mathrm{TiC}$ and amorphous carbon. The SnSb-TiC-C alloy shows an initial gravimetric capacity of $769 \mathrm{mAh} \mathrm{g}^{-1}\left(1385 \mathrm{mAh} \mathrm{cm}^{-3}\right)$, an initial coulombic efficiency of $84.9 \%$, and a tap density of $1.8 \mathrm{~g} \mathrm{~cm}^{-3}$. The buffer matrix of TiC and carbon in the nanocomposite alloy anodes accommodates the large volume change occurring during the charge-discharge process and leads to enhanced cyclability compared to pure $\mathrm{SnSb}$ anodes as well as previously published SnSb composites.
\end{abstract}

Keywords: lithium-ion batteries; alloy anodes; nanocomposites; mechanochemical reaction; electrochemistry

\footnotetext{
To whom correspondence should be addressed. Phone: 512-471-1791. Fax: 512-471-7681. E-mail: rmanth@mail.utexas.edu.
} 


\section{Introduction}

Due to their importance in energy storage for portable electronic devices as well as transportation, the performance enhancement of lithium-ion batteries has been a significant focus of research in recent years. An area of particular interest has been efforts to develop a lithium-ion anode material with higher capacity and enhanced safety characteristics relative to the currently used commercial graphite anode.

Lithium alloying (or lithium insertion) materials such as $\mathrm{Sn}$ and $\mathrm{Sb}$ have gained attention as potential candidates to meet this need due to their high theoretical capacities and higher operating potentials relative to $\mathrm{Li} / \mathrm{Li}^{+}$which grants greater safety from lithium plating during conditions of fast charge [1]. Both Sn and Sb have significantly higher theoretical capacities (991 and $660 \mathrm{mAh} \mathrm{g}^{-1}$, respectively) than graphite (372 $\mathrm{mAh} \mathrm{g}^{-1}$ ) because they form alloys with lithium that yield final compositions of, respectively, $\mathrm{Li}_{4.4} \mathrm{Sn}$ and $\mathrm{Li}_{3} \mathrm{Sb}$. This is much higher lithium content than allowed by the intercalation mechanism of graphitic anodes $\left(\mathrm{LiC}_{6}\right)$ [2,3]. Sn and $\mathrm{Sb}$ also react at average potentials of 0.6 and $0.9 \mathrm{~V}$ vs. $\mathrm{Li}_{\text {i }} \mathrm{Li}^{+}$which grants enhanced safety from lithium plating on the electrode surface and can limit degree of SEI layer formation, mitigating the negative effects of capacity loss and lower charge rate typically associated with SEI layer formation and growth [2-6].

The most significant issue preventing the implementation of these materials is a severely limited cycle life due to the very large volume change that occurs during the lithiation and delithiation processes. This large volume change results in lattice stress and crumbling of the active electrode particles, leading to rapid capacity fade [2,7-9]. One method to mitigate the negative effects of volume change is the incorporation of a reinforcing phase into the electrode to provide structural reinforcement and absorb some of the stress caused by the large active 
material volume change. The use of oxide phase active materials, such as $\mathrm{SnO}$ or $\mathrm{Sb}_{2} \mathrm{O}_{3}$, is one well-studied method to produce an electrode material with a finely incorporated reinforcing matrix, in this case an $\mathrm{Li}_{2} \mathrm{O}$ phase that is formed in situ during the first electrode lithiation cycle [1,9-12]. These oxide electrodes are able to operate with long cycle life and stable reversible capacity but suffer from a very large initial irreversible capacity due to the loss of active lithium with the formation of $\mathrm{Li}_{2} \mathrm{O}$, which makes them difficult to incorporate into practical battery systems $[1,9,11]$. Another method involves the use of intermetallic phase active materials, such as $\mathrm{Cu}_{6} \mathrm{Sn}_{5}$ or $\mathrm{FeSb}_{2}$, in which the reinforcing phase is again produced in situ during the lithiation of the electrode. However, in these materials the matrix is a metallic phase extruded from the intermetallic material that does not involve the robbing of active lithium from the system and therefore does not have the same issue of large initial capacity loss $[9,13]$. While these intermetallic materials exhibit cycle life enhancements they typically do not fully resolve the volume change losses and still have relatively short cycle lives [13-16]. A reinforcing phase can also be separately incorporated into the electrode material during the synthesis process such that it provides structural reinforcement with little or no interference to the electrochemical cycling process of the active material $[1,11]$. A few examples of such separately incorporated reinforcing phases are $\mathrm{TiC}, \mathrm{Al}_{2} \mathrm{O}_{3}$, and carbon incorporated into systems of metallic $\mathrm{Sn}$, metallic $\mathrm{Sb}$, or related intermetallics [17,22-24].

Nanoscale active materials have demonstrated enhanced cycling performance due to larger surface to volume ratios and shorter lithium ion diffusion distance which enhances the lithiation kinetics relative to micro-scale electrode materials [11-12,25-26]. In addition, nanoscale electrode materials are better able to accommodate the volume change of lithium alloying materials by limiting the build-up of internal stresses due to lattice mismatch during lithiation 
and delithiation. The incorporation of reinforcing phases into such nanoscale systems also provide the additional benefit of preventing particle agglomeration which would otherwise lead to particle growth and therefore end the enhancements provided by having nanoscale active material $[11,25]$.

The electrode composite analyzed in this paper is SnSb-TiC-C, composed of the active material $\mathrm{SnSb}$ with separately incorporated reinforcing phase of $\mathrm{TiC}$ and carbon. $\mathrm{SnSb}$ is an intermetallic alloy of two electrochemically active materials that reacts with lithium via two consecutive conversion reactions. A conversion reaction in an intermetallic anode material involves the extrusion of inactive materials from the alloy while the remaining active materials then react with lithium $[9,11]$. The reaction of $\mathrm{SnSb}$ with $\mathrm{Li}$ occurs in the two-step process shown below:

$$
\begin{gathered}
\mathrm{SnSb}+3 \mathrm{Li}^{+}+3 \mathrm{e}^{-} \rightarrow \mathrm{Li}_{3} \mathrm{Sb}+\mathrm{Sn} \\
\mathrm{Li}_{3} \mathrm{Sb}+\mathrm{Sn}+4.4 \mathrm{Li}^{+}+4.4 \mathrm{e}^{-} \rightarrow \mathrm{Li}_{3} \mathrm{Sb}+\mathrm{Li}_{4.4} \mathrm{Sn}
\end{gathered}
$$

The $\mathrm{Sb}$ reacts first (equation 1), as it has a higher reaction potential of $\sim 0.9 \mathrm{~V} v \mathrm{vs} . \mathrm{Li} / \mathrm{Li}^{+}$. The reaction of $\mathrm{Sn}$ (equation 2) occurs after the alloying of $\mathrm{Sb}$ and has multiple reaction potentials at $\sim 0.7 \mathrm{~V}$ and $\sim 0.4-0.2 \mathrm{~V}$ vs. $\mathrm{Li} / \mathrm{Li}^{+}[14,27]$. Because the reactions occur separately and consecutively, $\mathrm{Sn}$ and $\mathrm{Sb}$ alternatively act as the reinforcing and active phase in the intermetallic: initially $\mathrm{Sb}$ is lithiated while $\mathrm{Sn}$ acts as a stable reinforcing phase, then $\mathrm{Sn}$ lithiates while the formed $\mathrm{Li}_{3} \mathrm{Sb}$ is the reinforcing phase [14,26-31]. The separate reinforcing matrix of $\mathrm{TiC}$ in this composite electrode is selected for its high conductivity (resistivity $=0.3-0.8 \Omega \mathrm{cm}$ ) and structural integrity (knoop hardness $=2470 \mathrm{~kg} \mathrm{~mm}^{-2}$, compressive strength $=750-1300 \mathrm{MPa}$ ), 
which facilitate enhanced bulk electrode conductivity and greater accommodation of volume change strain [32]. The final electrode material of SnSb-TiC-C is formed using binary precursors of Ti-Sn and Ti-Sb which are put through a high energy mechanical milling (HEMM) process with carbon, yielding nanoparticulate $\mathrm{SnSb}$ intermixed with a $\mathrm{TiC}$ and carbon matrix. The resulting material is characterized with $\mathrm{x}$-ray diffraction (XRD), scanning electron microscopy (SEM), transmission electron microscopy (TEM), and electrochemical cycling.

\section{Experimental}

The SnSb-TiC-C composite was synthesized by first heating a mixture of metallic $\mathrm{Ti}$ (99.99\%, 325 mesh, Alfa Aesar) and metallic Sb (99.9\%, Aldrich), as well as a mixture of Ti and metallic Sn $\left(99.8 \%,<45 \mu \mathrm{m}\right.$, Aldrich) in a flowing argon atmosphere at $800{ }^{\circ} \mathrm{C}$ for $6 \mathrm{~h}$ to obtain Ti-Sb and Ti-Sn precursor materials. The Ti-Sb precursor contained an atomic ratio of Ti : $\mathrm{Sb}=2: 1$ and the Ti-Sn precursor contained the same atomic ratio of Ti $: \mathrm{Sn}=2: 1$. The Ti-Sn and $\mathrm{Ti}-\mathrm{Sb}$ precursors were then combined, mixed with carbon black, and subjected to highenergy mechanical milling (HEMM) to create a SnSb-TiC-C composite with 40 wt. \% SnSb, 40 wt. $\%$ TiC, and 20 wt. \% carbon black. The HEMM was carried out using Fritsch Pulverisette 6 with a stainless steel vial and milling balls. The sample was milled under an argon atmosphere at room temperature for $48 \mathrm{~h}$ at a speed of 500 RPM and with a ball to powder ratio of $20: 1$.

A $\mathrm{SnSb}-\mathrm{C}$ electrode material was also synthesized for comparative purposes. $\mathrm{SnSb}-\mathrm{C}$ was synthesized using a mixture of metallic $\mathrm{Sn}$ and $\mathrm{Sb}$ with an atomic ratio of $1: 1$ combined with 20 wt. \% carbon black and subjected to HEMM under similar conditions as described above.

The as-synthesized composite powder morphology was analyzed with a JEOL JSM-5610 SEM, with image capture and EDS elemental mapping, and a JEOL 2010F high-resolution TEM operating at $300 \mathrm{kV}$. The electrode powder was characterized by XRD with a Rigaku Miniflex 
600 with $\mathrm{Cu}-\mathrm{K} \alpha$ radiation. Tap density measurements were carried out on the as-synthesized powders using a Quantachrome AT-4 Autotap machine.

To analyze the electrochemical properties of $\mathrm{SnSb}-\mathrm{TiC}-\mathrm{C}$, electrodes were prepared by creating a slurry with 70 wt. \% active material $(\mathrm{SnSb}, \mathrm{TiC}$, and carbon are all considered to constitute the active material), 15 wt. \% Super P conductive carbon, and 15 wt. \% polyvinylidine fluoride (PVDF) binder dissolved in N-methyl pyrrolidone (NMP). Slurries were cast onto a $\mathrm{Cu}$ foil current collector using the doctor blade method and then dried for $24 \mathrm{~h}$ at $120^{\circ} \mathrm{C}$ in a vacuum oven. The electrodes were assembled in CR2032 coin cells in an Ar-filled glovebox with the cast electrode as the working electrode, lithium foil as the counter electrode, a Celgard polypropylene seperator, and $1 \mathrm{M} \mathrm{LiPF}_{6}$ in ethylene carbonate (EC) / diethyl carbonate (DEC) ( $1: 1 \mathrm{vol} / \mathrm{vol})$ as the electrolyte. The discharge / charge cycling experiments were performed at a constant current density of $100 \mathrm{~mA} \mathrm{~g}^{-1}$ on an Arbin battery cycler over a voltage range of 0 $2.0 \mathrm{~V}$ vs. $\mathrm{Li} / \mathrm{Li}^{+}$.

Ex situ SEM analysis was performed by first cycling the cells as described above. After the desired number of cycles was performed, the coin cell was opened in an argon filled glovebox and the electrode was extracted in the fully charged state. The sample was transported to the SEM in a sealed container and briefly exposed to air as it was transferred to the sample chamber of the JEOL JSM-5610 SEM for analysis.

\section{Results and Discussion}

Figure 1 shows the XRD patterns of the as-synthesized SnSb-C and SnSb-TiC-C powders. In Figure 1a, SnSb-C is shown to consist of the cubic stitstaite phase $\mathrm{SnSb}$ with a lattice constant of $a=5.88 \AA$ (JCPDS File: 00-026-0115). In Figure 1b, the SnSb-TiC-C pattern shows the same $\mathrm{SnSb}$ peaks along with additional peaks corresponding to crystalline TiC (cubic khamrabaevite 
phase) with a lattice constant of $a=4.33 \AA$ (JCPDS File: 00-031-1400). No peaks for carbon were seen in either sample, suggesting that it is amorphous within the electrode powder or mostly incorporated into the titanium carbide phase. In addition there are no other peaks corresponding to precursors or other phases, suggesting that $\mathrm{SnSb}$ and $\mathrm{TiC}$ are the only phases formed with no residual precursor material remaining. In order to measure and compare the active particle domain size in these two materials, the Scherrer equation was applied, which revealed an average crystal domain size of $11.7 \pm 0.7$ and $8.4 \pm 0.3 \mathrm{~nm}$, respectively, for $\mathrm{SnSb}-\mathrm{C}$ and SnSb-TiC-C.

SEM micrographs and EDS elemental mapping of the as-synthesized SnSb-TiC-C powder are shown in Figure 2. According to the high magnification image in Figure 2a, the powder appears to be composed of roughly spherical particles, with sizes ranging from approximately 20 $\mu \mathrm{m}$ to nanometer-scale particles. In Figure $2 \mathrm{~b}$ a lower magnification SEM image is overlaid with the composite elemental mapping of the four constituent elements of the composite electrode: $\mathrm{Sn}, \mathrm{Sb}, \mathrm{Ti}$, and $\mathrm{C}$. The individual elemental maps for these elements are also shown, respectively, in Figures 2c through $2 \mathrm{f}$. The individual and composite elemental mappings show all elements to be uniformly spread and intermixed throughout the electrode material.

Figure 3a shows a TEM image of a particle approximately $250 \mathrm{~nm}$ in size. The interior of the particle is composed of an amalgam of crystalline $\mathrm{TiC}$ and $\mathrm{SnSb}$ while the outer coating is carbon. HRTEM analysis in Figure $3 b$ shows this nano-scale crystalline morphology within the particle. Lattice spacing analysis confirms that both crystalline $\mathrm{SnSb}$ and $\mathrm{TiC}$ are finely intermixed in this particle yielding a highly desirable morphology of well-intermixed active and inactive phases that provides maximum protection from both active material volume change and agglomeration. This observation is in agreement with the elemental mapping data from Figure 2. 
The voltage profiles for the first five cycles of SnSn-TiC-C are shown in Figure 4. There is a noticeable irreversible capacity loss between the first and second discharge profiles occurring primarily at low voltages - below $\sim 0.4 \mathrm{~V}$. This irreversible loss can be attributed to the reduction of electrolyte on the electrode surface in the formation of an SEI layer. Subsequent cycles show very stable electrochemical performance with little change to curve shape or capacity. After the initial cycle SnSb-TiC-C has a sloping voltage curve on charge and discharge with no especially strong plateaus. Much of the reversible capacity comes from the reversible lithiation and delithiation of the $\mathrm{SnSb}$ active material with an average voltage upon discharge of $0.53 \mathrm{~V}$ and of $0.79 \mathrm{~V}$ upon charge.

The differential capacity plot (DCP) of the $\mathrm{SnSb}-\mathrm{TiC}-\mathrm{C}$ as well as similar systems is shown in Figure 5a-d. Figure 5a shows the DCP for an HEMM prepared electrode of active Sn and TiC. The $\mathrm{TiC}$ is considered as electrochemically inert though shows small reversible capacity at low voltages $\left(<0.2 \mathrm{~V}\right.$ vs. $\left.\mathrm{Li} / \mathrm{Li}^{+}\right)$in half-cell testing. A small peak corresponding to this $\mathrm{TiC}$ capacity is observable in Figure $5 \mathrm{a}$ and the rest of the peaks, which occur in the range of $\sim 0.7-$ $0.2 \mathrm{~V}$ on discharge and $\sim 0.35-0.85$ on charge, are attributable to the active Sn and match those expected from literature [7,14,27]. Figure 5b shows the DCP for an HEMM prepared Sb-C composite with the primary reaction peaks occurring from $\sim 1.0-0.7 \mathrm{~V}$ during discharge and $\sim$ $0.85-1.2 \mathrm{~V}$ during charging. These peaks also agree with those reported in literature for the lithiation and delithiation of $\mathrm{Sb}[2,6]$. The DCP for the intermetallic SnSb combined with carbon is shown in Figure 5c. In this DCP the reaction peaks previously correlated to the reactions of metallic Sn and Sb separately are both observable in the single electrode material. This is due to the reaction mechanism of $\mathrm{SnSb}$ which was explained previously in reactions 1 and 2 in which $\mathrm{Sn}$ and $\mathrm{Sb}$ essentially react independently in two sequential conversion reactions during 
lithiation. These observations are once again in agreement with literature on $\mathrm{SnSb}$ [14,26-31]. Figure 5d shows the DCP for the SnSb-TiC-C composite electrode material under investigation. The overall profile and peak locations are very similar to those observed with the SnSb-C sample in Figure $5 \mathrm{c}$, confirming that $\mathrm{SnSb}$ is indeed the primary active material in the composite. In addition, a sharp peak at low voltage could be attributable to the incorporation of $\mathrm{TiC}$ as observed with the Sn-TiC sample in Figure 5a. The primary difference between the SnSb-C and the $\mathrm{SnSb}-\mathrm{TiC}-\mathrm{C}$ plots is the broadening of peaks and loss of sharp reaction peaks for $\mathrm{SnSb}-\mathrm{TiC}-$ C. This is likely due to the smaller particle size of the active $\mathrm{SnSb}$ from the nano-scale incorporation of the reinforcing TiC phase shown in TEM analysis in Figure $3 \mathrm{~b}$ as confirmed previously with the Scherrer equation.

Figure 6 shows the cycle life of $\mathrm{SnSb}-\mathrm{TiC}-\mathrm{C}$ relative to a similar $\mathrm{SnSb}-\mathrm{C}$ composite electrode material. SnSb-TiC-C shows an initial discharge (lithiation) capacity of $769 \mathrm{mAh} \mathrm{g} \mathrm{g}^{-1}$ and an initial charge (delithiation) capacity of $653 \mathrm{mAh} \mathrm{g}^{-1}$. The capacity of the two different electrode materials is roughly equal for the first several cycles but the $\mathrm{SnSb}-\mathrm{C}$ electrode lacking the reinforcing TiC component suffers from a rapid capacity loss over the subsequent cycles, retaining a paltry capacity of $\sim 80 \mathrm{mAh} \mathrm{g}^{-1}(\sim 12 \%$ of its initial reversible capacity) after 100 cycles. The SnSb-TiC-C electrode demonstrates a markedly enhanced cycle life over SnSb-C, maintaining a capacity of $392 \mathrm{mAh} \mathrm{g}^{-1}$ (60\% of its initial reversible capacity) after 100 cycles. This cycle life enhancement is due to the reinforcing effect of the $\mathrm{TiC}$ that serves to mitigate the volume change effect in the electrode. In addition, the smaller particle size of the SnSb-TiC-C material likely serves to further limit the effects of the active material volume change during lithiation / delithiation of the electrode while the reinforcing $\mathrm{TiC}$ phase limits agglomeration to extend the effects of these small particle benefits. The cycle life enhancement can be further 
observed in Figure 7, where SEM images of the cycled and uncycled electrodes show that the $\mathrm{SnSb}$-TiC-C electrode is able to largely retain its structure and morphology after 50 cycles because the damaging effects of active-material volume change are mitigated.

The tap density of the as-synthesized SnSb-TiC-C electrode powder is $1.8 \mathrm{~g} \mathrm{~cm}^{-3}$, which yields an initial volumetric capacity for the material of $1385 \mathrm{mAh} \mathrm{cm}^{-3}$ during discharge and $1176 \mathrm{mAh} \mathrm{cm}^{-3}$ during charge. Figure 8 shows the volumetric cyclability performance of $\mathrm{SnSb}-$ TiC-C over 100 cycles as well as the coulombic efficiency of the electrode over those cycles. In the initial cycle $\mathrm{SnSb}-\mathrm{TiC}-\mathrm{C}$ shows a first cycle coulombic efficiency of $84.9 \%$. This is very high for an electrode utilizing a lithium alloying intermetallic active material as well as for a composite electrode structure [15-21,26,30]. The correspondingly low initial cycle irreversible capacity loss is $116 \mathrm{mAh} \mathrm{g}^{-1}$. After the initial cycle the coulombic efficiency increases to $>97 \%$ and by the $10^{\text {th }}$ cycle it is above $99 \%$. At this point the coulombic efficiency levels out and averages $~ 99.1 \%$ through 100 cycles. A coulombic efficiency of $>99.9 \%$ is generally desired for a battery electrode as it is expected to operate reversibly for hundreds of cycles, as such the capacity of SnSb-TiC-C is observed to fade but is able to retain a volumetric capacity of 705 $\mathrm{mAh} \mathrm{cm}^{-3}$ after 100 cycles. One potential cause of this observed capacity fade is an unstable SEI layer that cracks as the electrode undergoes volume change during cycling, exposing new electrode surface and resulting in continued capacity loss [2,33]. There is potential to further mitigate this fade with electrolyte additives like fluoroethylene carbonate and vinylene carbonate to stabilize the SEI layer during cycling [34]. This extended cycle life is an improvement upon bulk $\mathrm{SnSb}$ anodes and is comparable to the extended cycling performance of previously published $\mathrm{SnSb} / \mathrm{C}(60 / 40)$ anodes while offering the benefit of high tap density and correspondingly high volumetric capacity as well [14,26-31]. 


\section{Conclusion}

A SnSb-TiC-C nanocomposite anode has been synthesized via a HEMM method and characterized by XRD, SEM, and TEM. This characterization revealed that the SnSb-TiC-C sample is composed of nanoscale particles of crystalline $\mathrm{SnSb}$ well incorporated with a reinforcing matrix of crystalline $\mathrm{TiC}$ and amorphous $\mathrm{C}$. The SnSb-TiC-C composite exhibits significantly enhanced cyclability relative to similarly prepared $\mathrm{SnSb}-\mathrm{C}$, retaining a capacity of $392 \mathrm{mAh} \mathrm{g}^{-1}$ after 100 cycles while the $\mathrm{SnSb}-\mathrm{C}$ composite retains almost no capacity $(\sim 80 \mathrm{mAh}$ $\left.\mathrm{g}^{-1}\right)$. The material also exhibits a very high initial coulombic efficiency of $84.9 \%$ and a tap density of $1.8 \mathrm{~g} \mathrm{~cm}^{-3}$. These are practically useful metrics as they allow for, respectively, greater total electrode usage in full cells and greater volumetric energy density. Due to its large capacity, enhanced cycle life, high tap density, and high first cycle coulombic efficiency, SnSb-TiC-C offers an electrode system with great potential for future development and application as a lithium ion battery anode.

\section{Acknowledgements}

This work was supported by the U.S. Department of Energy, Office of Basic Energy Sciences, Division of Materials Sciences and Engineering, under award number DE-SC0005397. 


\section{References}

[1] J.-M. Tarascon, M. Armand, Nature 414 (2001) 359-367.

[2] W.-J. Zhang, J. Power Sources 196 (2011) 13-24.

[3] A. Manthiram, J. Phys. Chem. Lett. 2 (2011) 176-184.

[4] J. B. Goodenough, Y. Kim, Chem. Mater. 22 (2010) 587-603.

[5] G. Derrien, J. Hassoun, S. Panero, B. Scrosati, Adv. Mater. 19 (2007) 2336-2340.

[6] C. M. Park, S. Yoon, S.I. Lee, J. H. Kim, J. H. Jung, H. J Sohn, J. Electrochem. Soc. 154 (2007) A917-A920.

[7] M. Winter, J. O. Besenhard, Electrochim. Acta 45 (1999) 31-50.

[8] U. Kasavajjula, C. Wang, A.J. Appleby, J. Power Sources 163 (2007) 1003-1039.

[9] C.-M Park, J.-H Kim, H. Kim, H.-J Sohn, Chem. Soc. Rev. 39 (2010) 3115-3141.

[10] R. Retoux, T. Brousse, D.M. Schleich, J. Electrochem. Soc. 146 (1999) 2472-2476.

[11] L. Ji, Z. Lin, M. Alcoutlabi, X. Zhang, Energy Environ. Sci. 4 (2011) 2682-2699.

[12] N. Li, C. R. Martin, B. Scrosati, Electrochem. Solid-State Lett. 3 (2000) 316-318.

[13] K.D. Kepler, J.T. Vaughey, M.M. Thackeray, J. Power Sources $81-82$ (1999) 383-387.

[14] C.-M. Park, H.-J. Sohn, Electrochim. Acta 54 (2009) 6367-6373.

[15] C.Q. Zhang, J.P. Tu, X.H. Huang, Y.F. Yuan, S.F. Wang, F. Mao, J. Alloys Compd. 457 (2008) 81-85.

[16] J. Yin, M. Wada, S. Yoshida, K. Ishihara, S. Tanase, T. Sakaia, J. Electrochem. Soc. 150 (2003) A1129-A1135.

[17] D. Applestone, A. Manthiram, RSC Advances 2 (2012) 5411-5417.

[18] S. Yoon, A. Manthiram, J. Mat. Chem. 20 (2010) 236-239.

[19] E. Allcorn, A. Manthiram, J. Phys. Chem. C 118 (2014) 811-822. 
[20] E. Allcorn, A. Manthiram, ACS Appl. Mater. Interfaces 6 (2014) 10886-10891.

[21] D. Applestone, S. Yoon, A. Manthiram, J. Mat. Chem. 22 (2012) 3242-3248.

[22] S. Yoon, A. Manthiram, Chem. Mater. 21 (2009) 3898-3904.

[23] I. T. Kim, E. Allcorn, A. Manthiram, Phys. Chem. Chem. Phys. 16 (2014) 12884.

[24] I. T. Kim, S. O. Kim, A. Manthiram, J. Power Sources 269 (2014) 848.

[25] K. E. Aifantis, S. A. Hackney, J. P. Dempsey, J. Power Sources 165 (2007) 874-879.

[26] F. Wang, M. Zhao, X. Song, J. Alloys Compd. 472 (2009) 55-58.

[27] J. Yang, Y. Takeda, N. Imanishi, J.Y. Xie, O. Yamamoto, Solid State Ionics 133 (2000) 189-194.

[28] M.S. Park, S. A. Needham, G. X. Wang, Y. M. Kang, J. S. Park, S. X. Dou, H. K. Liu, Chem. Mater. 19 (2007) 2406-2410.

[29] L. Simonin, U. Lafont, E. M. Kelder, J. Power Sources 180 (2008) 859-863.

[30] C.-M. Park, K.-J. Jeon, Chem. Commun. 47 (2011) 2122-2124.

[31] H. Li, L. Shi, W. Lu, X. Huang, L. Chen, J. Electrochem. Soc. 148 (2001) A915-A922.

[32] J. Shackelford, CRC Practical Handbook of Materials Selection, second ed., CRC Press, Boca Raton, FL, 1995.

[33] M. Wachtler, J. O. Besenhard, M. Winter, J. Power Sources 94 (2001) 189-193.

[34] D. Applestone, A. Manthiram, J. Power Sources 217 (2012) 1-5. 


\section{Figure Captions}

Figure 1. XRD of as-synthesized (a) $\mathrm{SnSb}-\mathrm{C}$ and (b) $\mathrm{SnSb}-\mathrm{TiC}-\mathrm{C}$ with $\mathrm{SnSb}$ and $\mathrm{TiC}$ peaks identified.

Figure 2. (a) SEM image of SnSb-TiC-C as-sythesized electrode powder showing roughly spherical particles with sizes ranging from roughly 20 microns to the nanometer scale and (b) a lower magnification SEM image with overlaid combined EDX results for $\mathrm{Sn}, \mathrm{Sb}, \mathrm{Ti}$, and $\mathrm{C}$. The individual elemental EDX results are also shown for (c) Sn, (d) Sb, (e) Ti, and (f) C.

Figure 3. TEM images of SnSb-TiC-C as-synthesized electrode powder. (a) Low magnification image with diffraction pattern confirming crystalline phases. (b) High magnification image showing lattice fringes matched to $\mathrm{SnSb}$ and $\mathrm{TiC}$ phases.

Figure 4. Voltage vs. capacity plot of the first 5 cycles of an SnSb-TiC-C electrode.

Figure 5. Differential capacity plots (DCP) of (a) Sn-TiC composite demonstrating the reaction peaks of $\mathrm{Sn}$ (marked in red box) and $\mathrm{TiC}$, (b) $\mathrm{Sb}-\mathrm{C}$ composite showing the reaction peaks of $\mathrm{Sb}$ (marked in blue dashed box), (c) SnSb-C showing the combined reaction peaks of $\mathrm{Sn}$ and $\mathrm{Sb}$, and (d) SnSb-TiC-C.

Figure 6. Cyclability comparison of SnSb-TiC-C and SnSb-C electrodes over 100 cycles.

Figure 7. Ex situ SEM of the surface of an SnSb-TiC-C electrode (a) before cycling and (b) after 50 cycles.

Figure 8. Volumetric capacity and coulombic efficiency of SnSb-TiC-C over the first 100 cycles. 
Figures

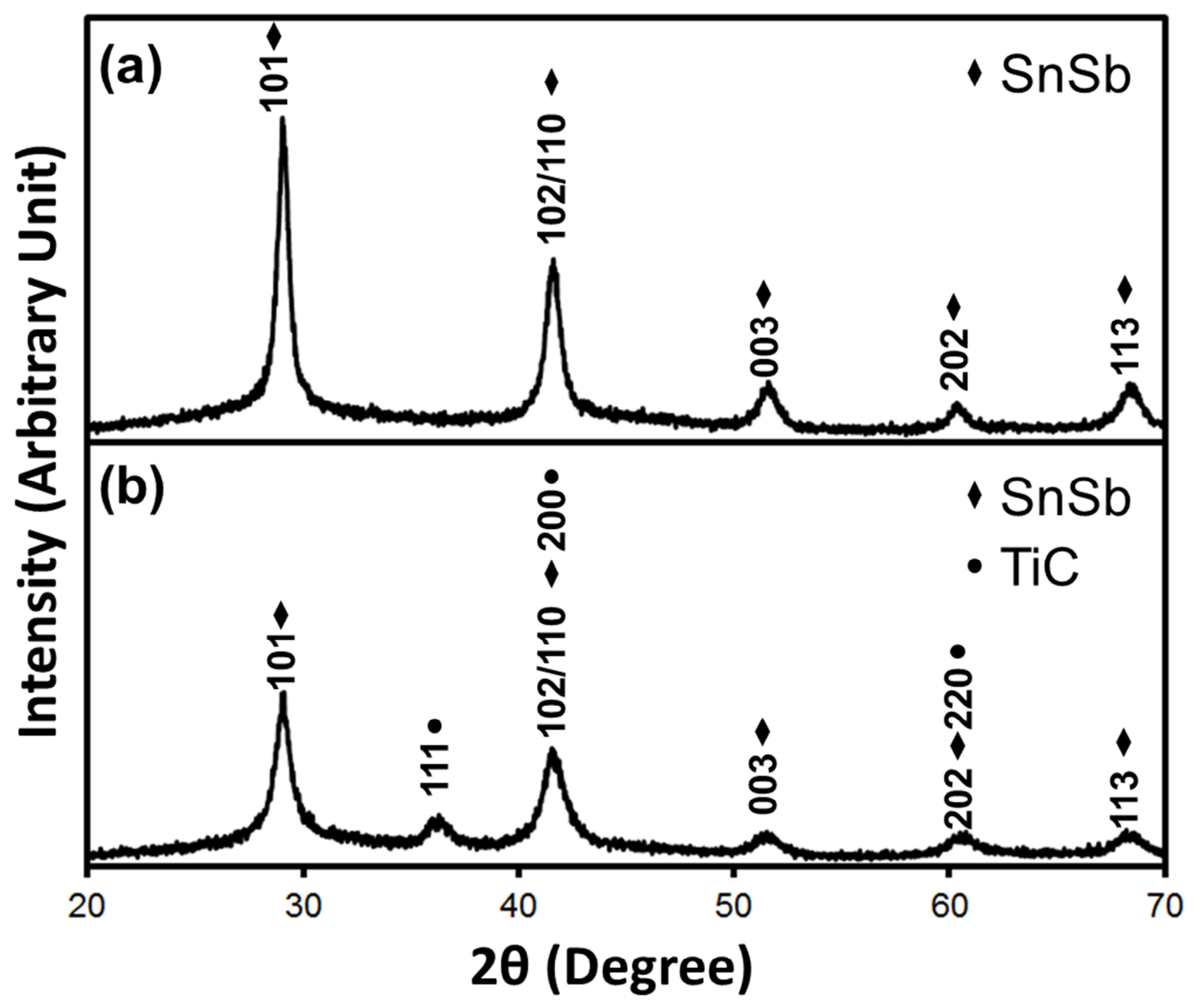

Figure 1 

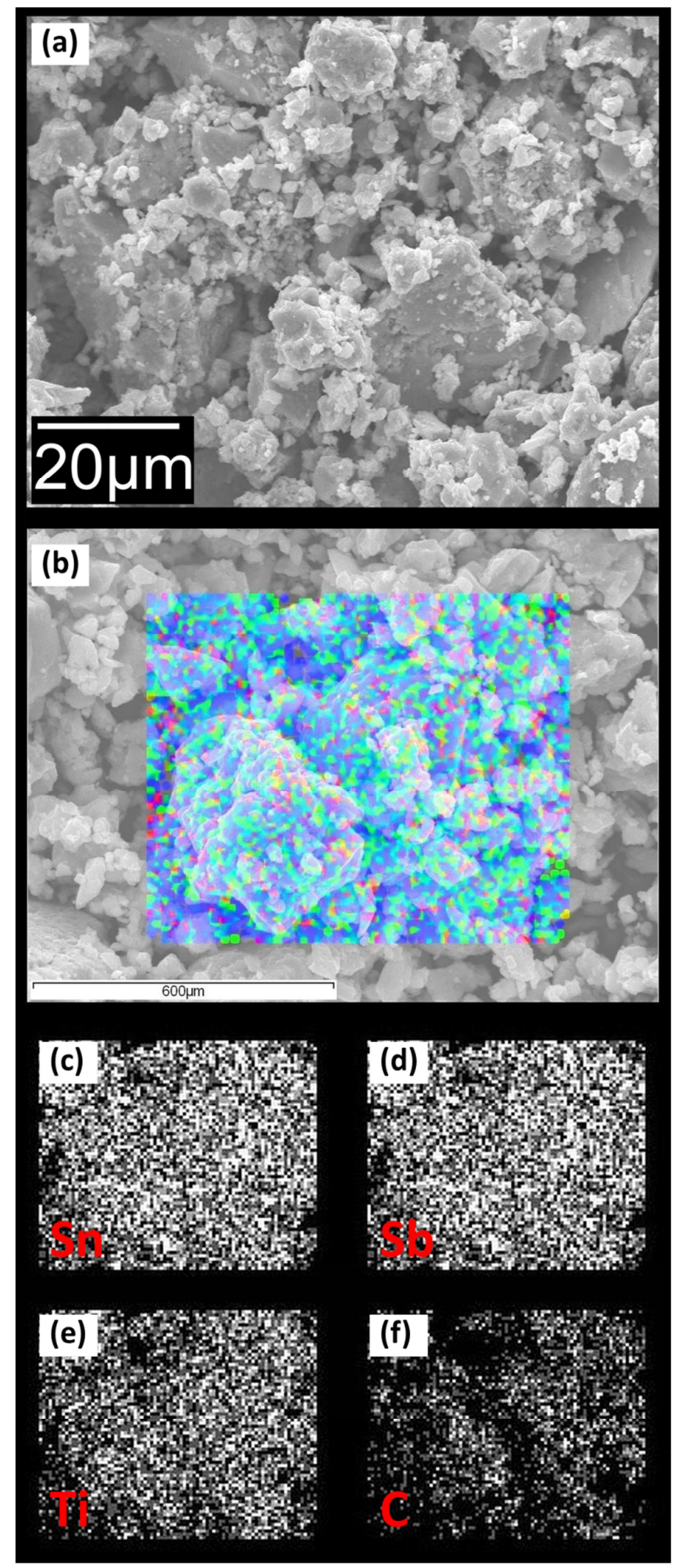

Figure 2 


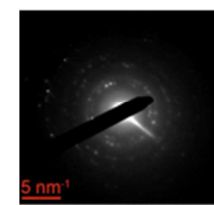

(a)

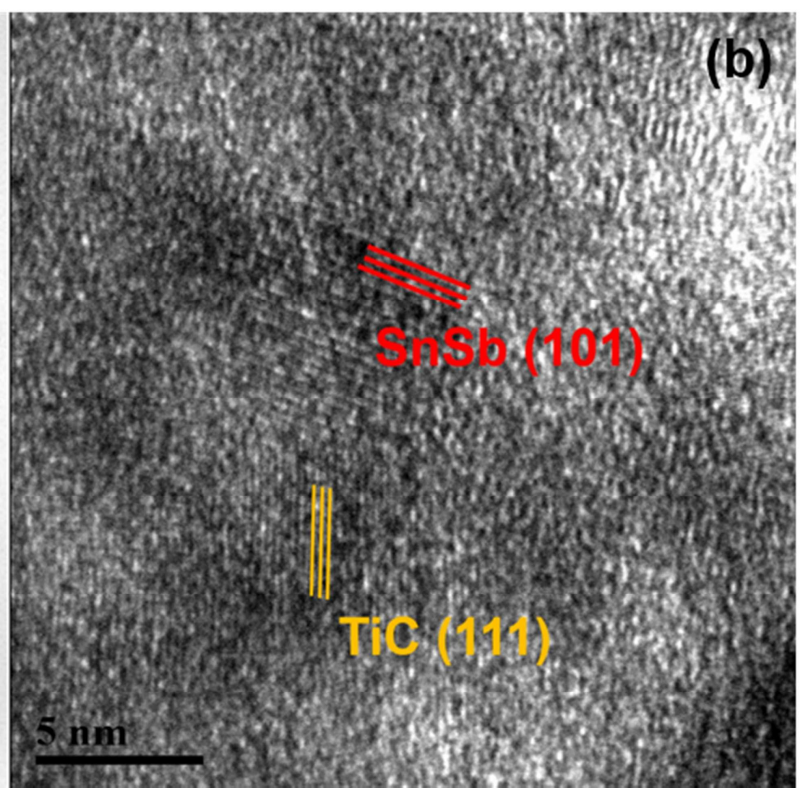

Figure 3 


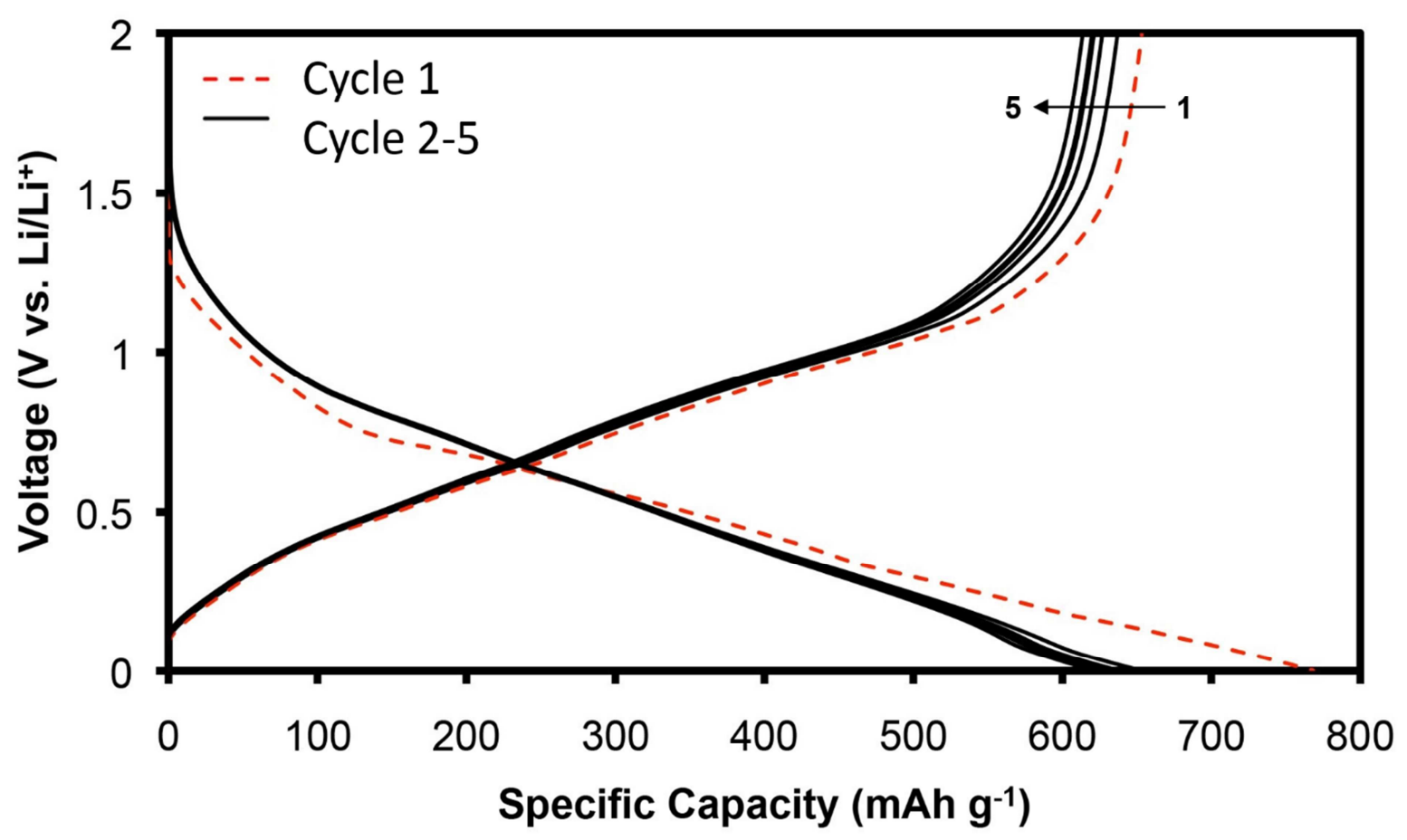

Figure 4 


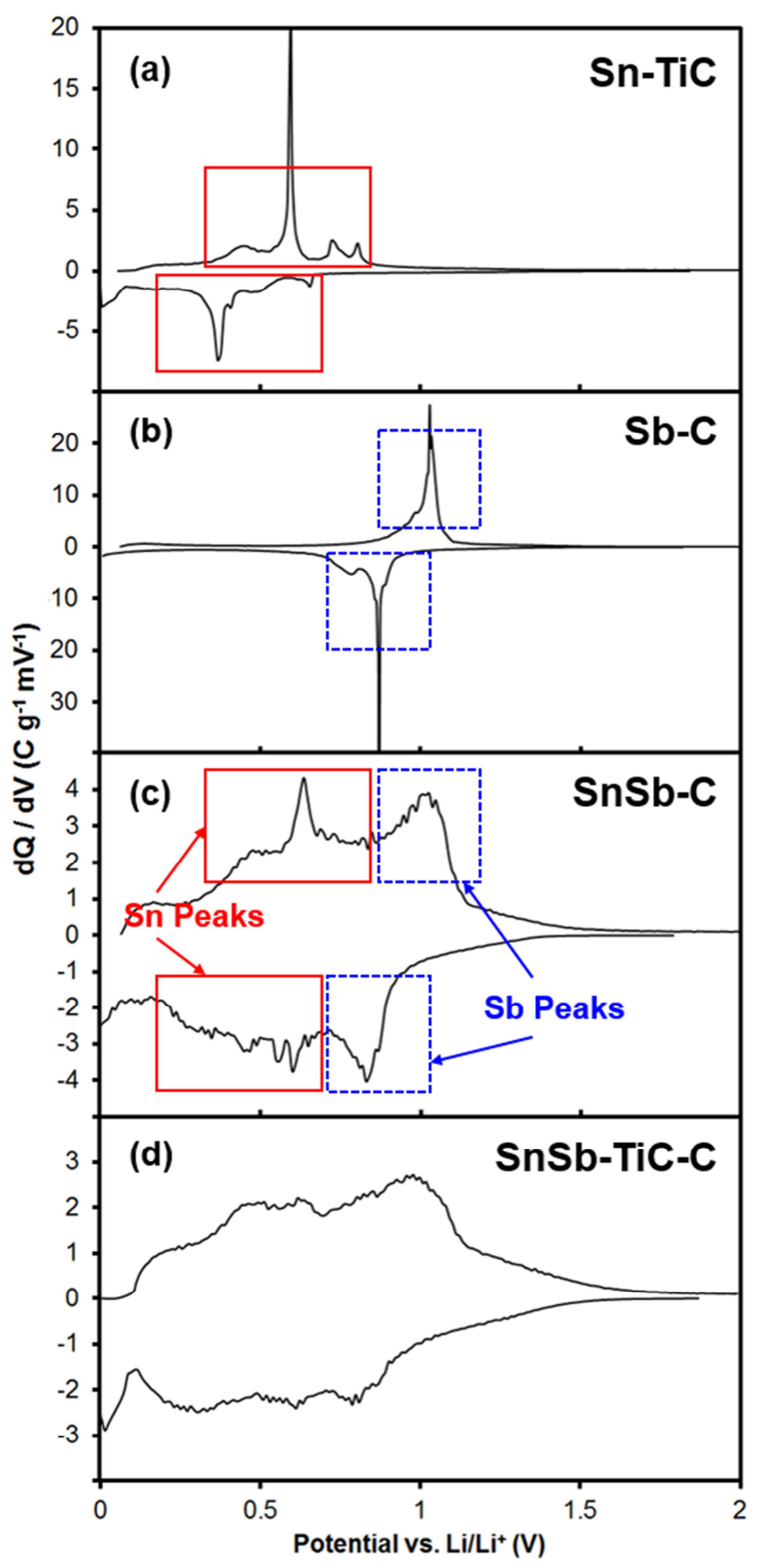

Figure 5 


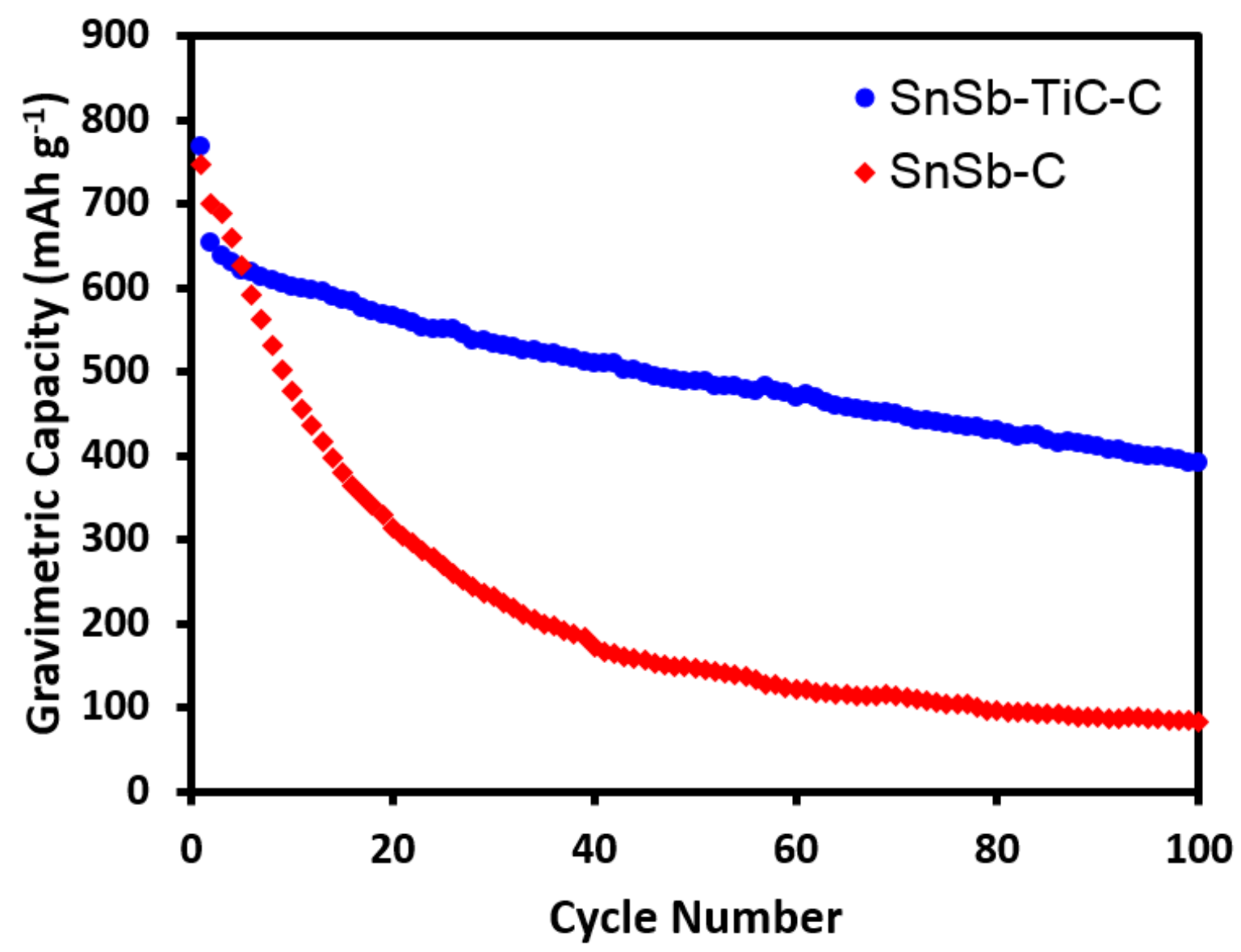

Figure 6 


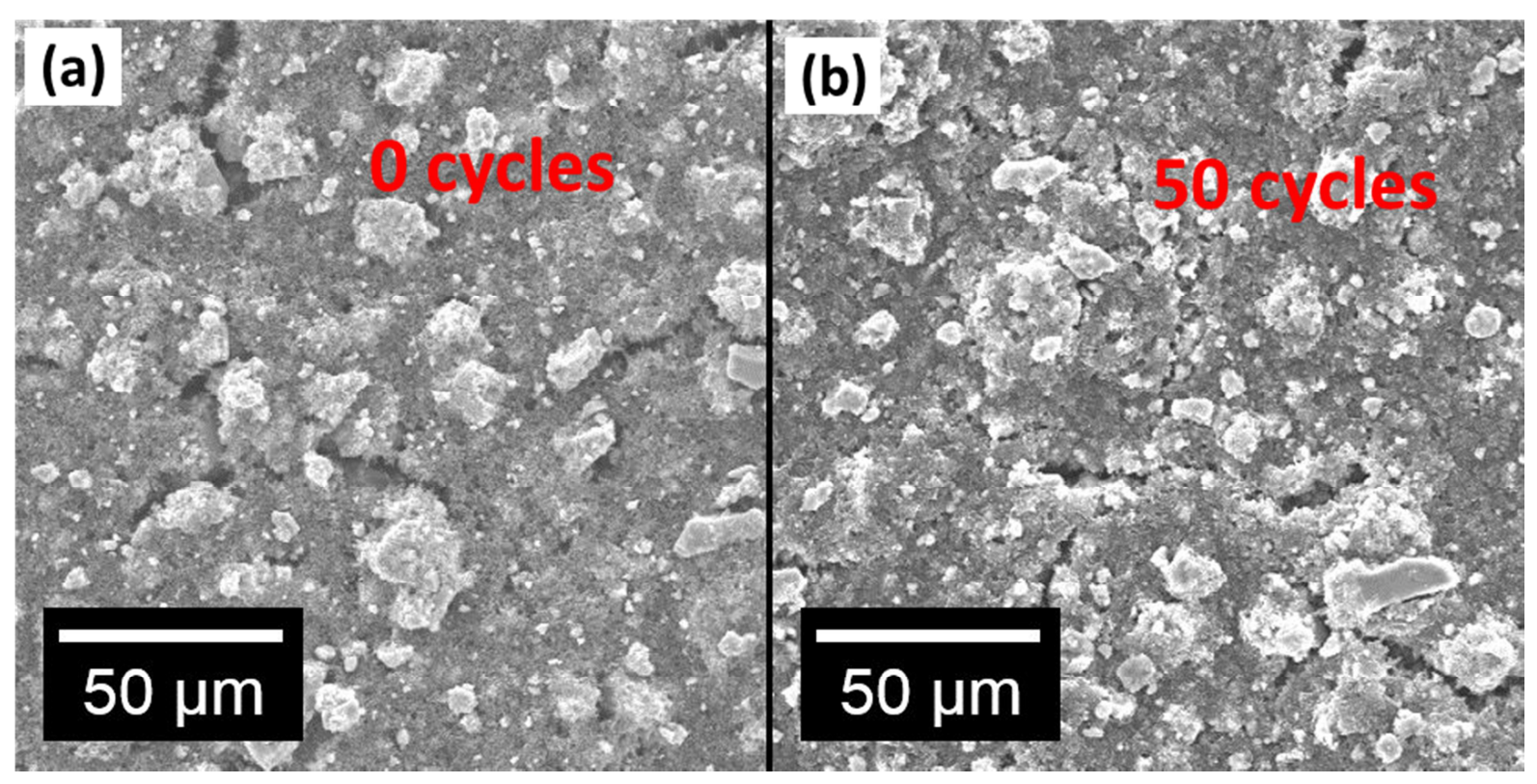

Figure 7 


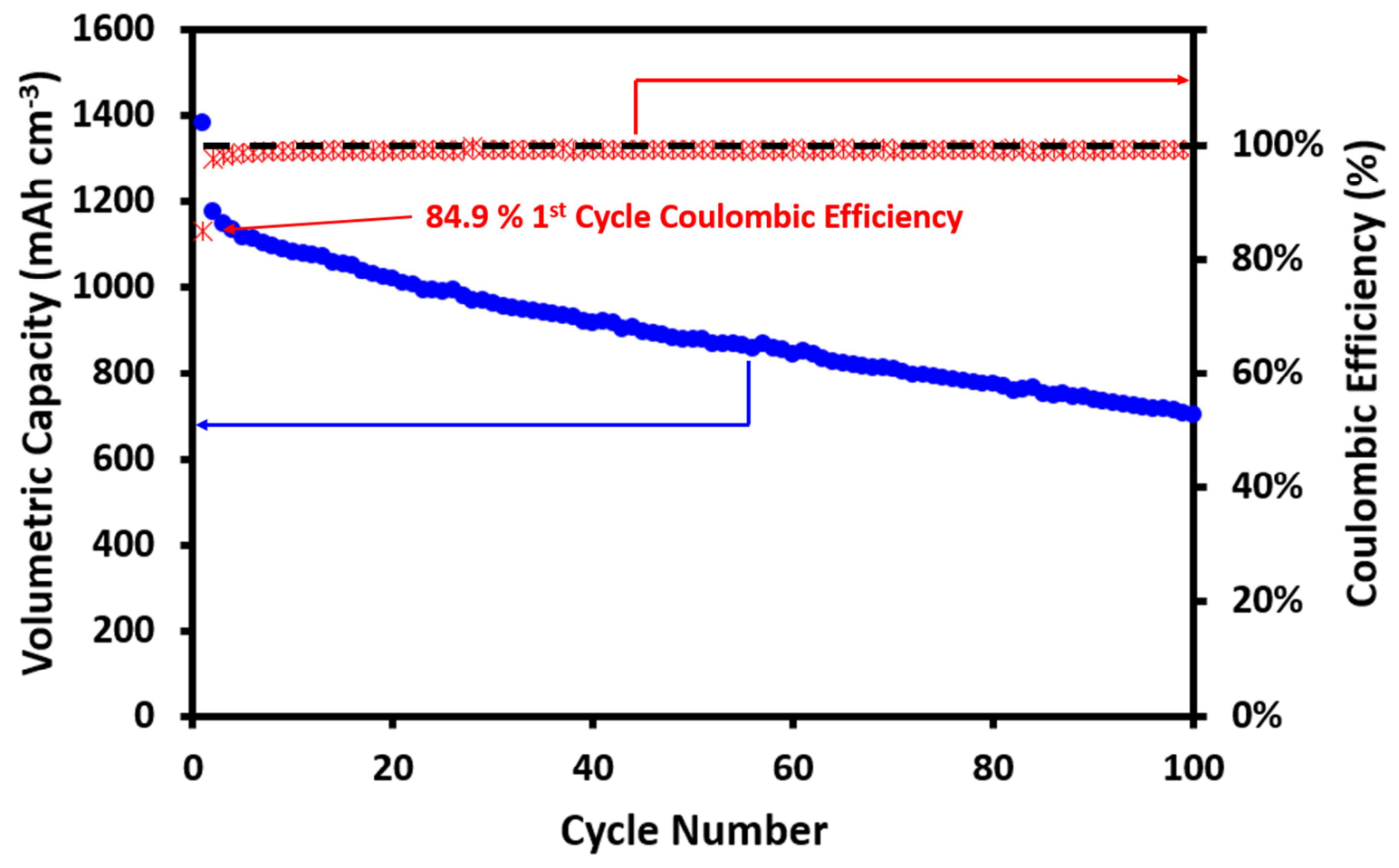

Figure 8 